\title{
O álcool e os jovens
}

\author{
RUI ANTÓNIO ROCHA TATO MARINHO*
}

\section{RESUMO}

O consumo excessivo de álcool é uma ameaça à saúde pública mundial segundo a Organização Mundial de Saúde (OMS). 0 consumo de risco está a generalizar-se na juventude europeia. Um quarto dos jovens europeus dos 15 aos 29 anos morre devido ao álcool em excesso. Portugal é uma dos países de maior consumo mundial. Assiste-se ao início do consumo em idades muito precoces (13 anos), na generalização do consumo excessivo nas raparigas, e na adopção muito frequente do consumo tipo "binge drinking» (bebedeira, embriaguez). Este tem consequências muito graves.

O álcool provoca cerca de 60 doenças, sendo as de maior impacto em Portugal a cirrose alcoólica e as mortes por acidentes de viação, estas particularmente nos jovens. A cirrose hepática é a décima causa de morte e cerca de 2/3 são de etiologia alcoólica. As mortes por acidentes de viação são de longe a principal causa de morte nos jovens portugueses. Noventa por cento dos acidentes mortais estão relacionados com o factor humano e estima-se que em metade o álcool em excesso esteja na origem do acidente mortal.

As medidas a tomar para reduzir os danos do consumo de álcool devem assentar na informação. No entanto, para se fer resultados em termos imediatos, na vertente de evitar algumas mortes, a repressão do consumo de álcool associado à condução nos jovens, não só no aspecto preventivo (aumentar a frequência do teste do balão), como também punitivo (aplicacão efectiva e exemplar da lei) é a medida comprovadamente mais eficaz. Estas medidas permitiram salvar milhares de vidas nos países onde têm sido aplicadas, como por exemplo no Reino Unido.

Palavras Chave: Jovens; Álcool; Binge Drinking; Sinistralidade atribuiveis aos efeitos adversos da ingestão excessiva de álcool. Nalguns países da Europa de Leste, aquele número chega a atingir um terço e reduziu a esperança média de vida, principalmente no sexo masculino, em 10 a 12 anos. Pelo contrário, a percentagem mundial de jovens dos 15 aos 29 anos que morrem por causa do álcool é de 5\%.

Estima-se que mais do que 2,3\% da população mundial seja alcoólica o que corresponde a cerca de 140 milhões. Portugal é o sétimo consumidor de álcool de forma global e o quarto consumidor mundial de vinho (World Drink Trends, 2004) (Figura 1 e 2). Sendo um dos maiores consumidores mundiais, o problema é particularmente grave e os números apontam para que possam existir em Portugal cerca de 600.000 alcoólicos e 750.000 bebedores excessivos.

Em Portugal a cirrose hepática, uma das consequências mais graves do alcoolismo, é a décima causa de morte, tendo sido responsável por 1.526 mortos em 2005 (Instituto Nacional de Estatística). Cerca de dois terços destas cirroses são de etiologia alcoólica. ${ }^{1}$ Este número é de certeza inferior ao real. Com efeito morreram no mesmo ano 329 portugueses por carcinoma hepatocelular. Este tumor está, na Europa, praticamente sempre associado à cirrose hepática. Pensa-se que o álcool poderá estar na sua etiologia em cerca de 20 a 40\% dos casos, de forma isolada ou como co-factor. Morreram por SIDA 876 indivíduos em 2005, mas cerca de $25 \%$ por cirrose hepática no contexto de co-infecção VIH com o vírus da hepatite $\mathrm{C}$. $\mathrm{O}$ consumo de álcool é nestes casos também um factor agravante da evolução da cirrose.

O álcool está também fortemente as- 


\section{DOSSIER}

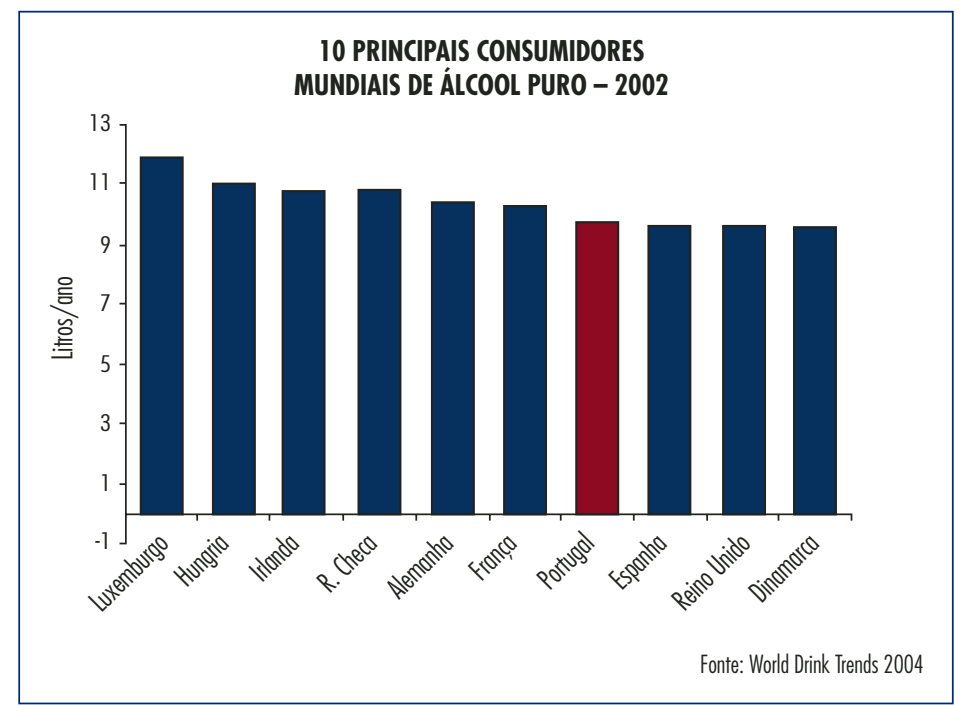

Figura 1. Portugal encontra-se dentro dos 10 maiores consumidores de álcool a nível mundial (fonte World Drink Trend, 2004).

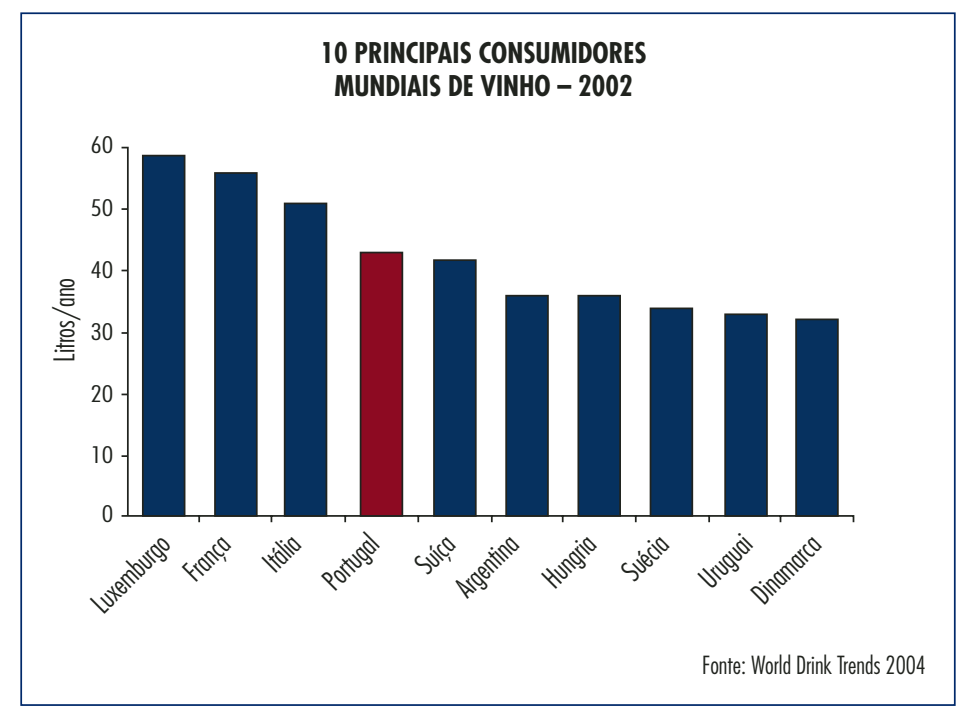

Figura 2. Portugal é $04^{\circ}$ consumidor mundial de vinho. (fonte World Drink Trend, 2004).

sociado às mortes na estrada nos jovens portugueses. Portugal tem tido, apesar da evolução favorável nos últimos anos, um dos piores índices europeus de sinistralidade rodoviária. Ora, 90\% dos acidentes rodoviários mortais são causados por erro humano, dos quais cerca de $40-50 \%$ associados ao excesso de álcool.

\section{O ÁlcoOl É UMA Droga?}

Sim, é uma droga apesar de lícita. Provoca dependência física e psíquica (habituação). O alcoolismo é considerado uma doença, muito incapacitante, de implicações sociais graves, mas tratável. 


\section{Que Custos?}

Nos países da União Europeia, os problemas derivados do consumo excessivo atingem 5-6\% dos Produtos Nacionais Brutos (PNB), número superior à contribuição da produção de bebidas alcoólicas para o mesmo PNB, que é de $2 \%$.

\section{Quals as Doencas Provocadas PELO ÁlCOOL?}

O consumo excessivo de álcool pode levar classicamente a 61 doenças: figado (hepatite alcoólica, esteatose, cirrose, cancro do figado), acidentes de viação mortais (na origem de $40 \%$ dos acidentes em Portugal), $1 / 3$ dos suicídios, $1 / 3$ dos homicídios, 1/3 dos afogamentos, impotência (afecta 25\% dos alcoólicos), infertilidade, hipertensão arterial, síndroma alcoólico fetal (atraso mental, cardiopatia, alterações faciais), dificuldade de aprendizagem, mau rendimento escolar, depressor do Sistema Nervoso (cérebro, cerebelo), hemorragia subdural, alterações do comportamento (psicoses, encefalopatias), depressão, ansiedade, anemia, cancro do esófago, faringe, boca, laringe, figgado, pâncreas, polineuropatias, úlcera péptica, gastrite, varizes no esófago, pancreatite aguda e crónica, miocardiopatia, miopatia, violações, violência doméstica (40-70\%), quedas acidentais, fogos postos, acidentes de trabalho (quedas em altura, por exemplo), perda de produtividade, absentismo, sexo desprotegido (co-factor na epidemiolgia da transmissão do VIH), insucesso escolar, esquizofrenia, etc.

Quanto mais se bebe, maior o risco. ${ }^{2}$

\section{E o factor Protector Cardíaco?}

A importância cultural e social do con- sumo de álcool não pode ser ignorada. Contudo, a OMS, baseada em diversos estudos científicos, no que diz respeito à eventual protecção cardiovascular conferida pela ingestão de álcool, não aceita que o seu consumo seja promovido concluindo (já em 1984) que "Increased alcohol intake is not recommended as a preventive measure in coronary heart disease, either in populations or individuals, WHO Expert Committee on the Prevention of Coronary Heart Diseasen. ${ }^{3}$ A mesma opinião é partilhada pela International Task Force for Prevention of Coronary Heart Disease (www.chdtaskforce.de/guidelines/kap28.htm). Segundo estas entidades, consumo moderado equivale à ingestão de cerca de 30 gramas de álcool por dia, 40 gramas no homem e 20 gramas na mulher (aproximadamente duas bebidas no homem e uma na mulher). Quantidades superiores são excessivas. ${ }^{4}$ A OMS refere ainda limites mais baixos: no homem, 24 gramas de álcool $\left(250 \mathrm{~cm}^{3} \mathrm{de}\right.$ vinho a $12^{\circ}$ ou três "imperiais»); na mulher, 16 gramas de álcool $\left(150 \mathrm{~cm}^{3} \mathrm{de}\right.$ vinho ou duas "imperiais»).

\section{EXISTE RISCO DE CANCRO?}

Sim, beber álcool em excesso provoca cancro: do figado, do esófago, da boca, garganta, mama. Pensa-se que também possa estar implicado no cancro do cólon. O álcool é considerado pela OMS como um agente carcinogénico.

\section{A Partir de Que Idade SE Pode Beber?}

Não se deve beber antes dos 18 anos. Isto apesar da idade legal em Portugal ser de 16 anos. Consumir antes dos 18 aumenta o risco de dependência. Existem estudos que demonstram que $40 \%$ dos jovens que começam a beber de forma excessiva aos 13 anos ficam dependentes do álcool. ${ }^{5}$ Os portugueses come- 
çam a beber muito cedo, em média aos 13 anos.

\section{Qual a Dose Segura por Dia, POR OCASIÃO FESTIVA?}

Os homens não devem beber mais do que duas a três por dia e cinco em ocasiões festivas. As mulheres não mais do que duas a três por dia e quatro em ocasiões festivas (consumo num curto espaço de tempo). Um dos fenómenos mais graves em Portugal e na Europa é a chamada feminização do alcoolismo, ou seja as raparigas, pelo menos nas bebidas destiladas, estão a beber tanto como os rapazes.

A morte por cirrose alcoólica, nas mulheres jovens inglesas aumentou cerca de $1000 \%$ nos últimos 30 anos.

\section{As BEbedeIRAs (BINGE DRINKING) FAZEM MAL?}

Sim, e muito. Além do risco de acidente de viação, existe o risco de violência (homicídios, roubos, violência sexual), quedas, sexo não seguro. ${ }^{6}$ Além disso, as células cerebrais podem ficar danificadas de forma irreversivel.

$\mathrm{O}$ risco de ter sexo não seguro (não previsto, sem protecção, com desconhecidos, sexo em grupo, muitas vezes sem recordação no dia seguinte) aumenta $700 \%$.

Aproximadamente 90\% dos assassínios múltiplos nos estabelecimentos escolares (EUA) estão relacionados com o excesso de consumo de álcool.

\section{O Álcool Mata na Estrada?}

De forma muito evidente. É um problema grave de segurança interna que Portugal tem enfrentado. $\mathrm{O}$ acidente de viação é, a grande distância, a causa principal de morte nos jovens dos 1 aos 29 anos (Figura 3). Por exemplo, em 2005 em Portugal morreram pelo menos 471 jovens nas estradas, 65 por SIDA e 171 por cancro.

Atentem-se nestes números: de 1991 a 2001 em Portugal morreram 27.167 portugueses em acidentes de viação (em 10 milhões de habitantes). No mesmo período morreram na Suécia $6.660 \mathrm{Su}$ ecos em acidentes de viação $(9,2$ milhões de habitantes) e 42.167 no Reino Unido (59 milhões de habitantes)!

Os acidentes de viação, em termos de morbilidade, mortalidade e impacto sócio-económico, têm uma amplitude muito superior a algumas doenças oncológicas. Devem ser entendidos como uma verdadeira "doença grave», felizmente susceptivel de prevenção.

Diversos estudos têm demonstrado que o factor humano (erro humano na percepção devido principalmente a excesso de velocidade e ao consumo de álcool) é o primeiro responsável em 80$-90 \%$ dos acidentes. ${ }^{7}$ Com efeito, o consumo de álcool aumenta não só o risco de acidente, bem como a sua gravidade e consequentemente a probabilidade de ocorrência de mortos.

$\mathrm{O}$ acto da condução implica a execução de múltiplas e sucessivas tarefas de forma contínua e continuada que se alteram muitas vezes de forma inesperada (travão, acelerador, embraiagem, volante, mudanças, espelho retrovisor, espelhos laterais, atenção à estrada, ao velocímetro, ao nivel de gasolina, à sinalização, aos painéis publicitários, outros veículos, peões, além do rádio, cinzeiro, relógio, telemóvel, GPS, conversação com outros passageiros, etc).

Calcula-se que, e de acordo com a média europeia, ${ }^{8} 25-30 \%$ das mortes dos condutores estão claramente associadas a Taxas de Alcoolemia no Sangue (TAS) excessivas. Esse número nos Estados Unidos da América (EUA) é de 40\% (2000), ${ }^{9}$ França 50\%, Canadá $39,1 \%$, Chile $42 \%$, Reino Unido $32 \%$ e 


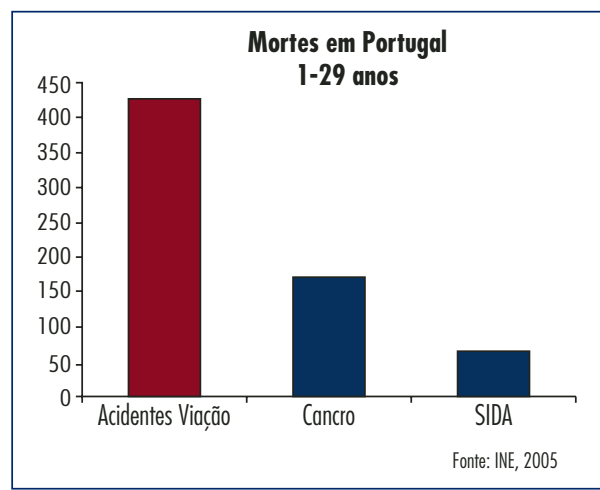

Figura 3. Principais causas de morte em Portugal dos 1-29 anos (fonte Instituto Nacional de Estatística, 2005)

no Brasil 50,6\%. ${ }^{10}$

A actuação eficaz das forças de segurança, no controlo da velocidade e do álcool, tem salvo milhares de vidas em todo o Mundo. É curioso e importante reparar nas conclusões do estudo publlicado no Lancet em 2003: Traffic-law enforcement effectively reduces the frequency of fatal motor-vehicle crashes in countries with high rates of motor-vehicle use. Inconsistent enforcement, therefore, may contribute to thousands of deaths each year worldwide. ${ }^{11}$

A condução sob o efeito do álcool é considerada pela UE como uma prioridade-chave da segurança rodoviária. ${ }^{12}$ A «Carta Europeia sobre o Consumo do Álcool" da responsabilidade da OMS (1995), composta por 10 pontos referentes à estratégia de luta contra o alcoolismo, recomendava textualmente no seu ponto 3 "promulgar e reforçar leis que desencorajem, com eficácia, a condução sob o efeito do álcool". ${ }^{13}$ As estimativas da UE apontam para que, pelo menos, 10.000 vidas poderiam ser poupadas caso fosse eliminada a condução sob o efeito do álcool.

O valor social desta perda de vidas, utilizando a regra de 1 milhão de euros, formulada no Programa de Segurança Rodoviária da UE, eleva-se a 10.000 milhões de euros por ano. Se cada vida "valesse» 1 milhão de Euros (valor atri-

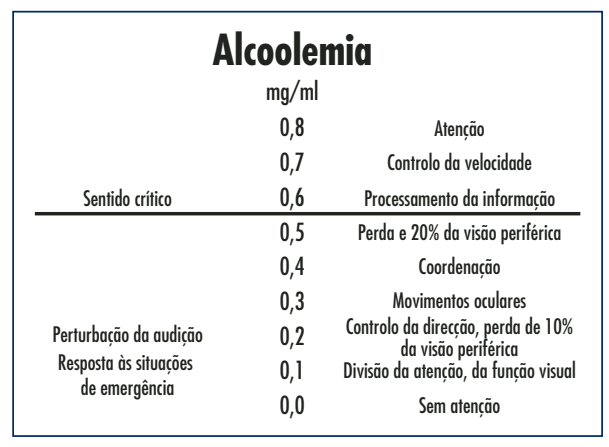

Figura 4. Alteração das capacidades de coordenação já com 0,1 a $0,2 \mathrm{mg} / \mathrm{ml}$.

buído nalguns estudos), se bem que a vida obviamente não tem preço...

\section{Que É a Alcoolemia? 0 Que} SIGNIFICA 0,2 MG/ML, 0,5 MG/ML?

Alcoolemia significa a quantidade de álcool medida no sangue. Cada bebida que se consome, sobe em média a alcoolemia em 0,2 mg/ml. O limite legal em Portugal é de $0,5 \mathrm{mg} / \mathrm{ml}$. O risco de acidente mortal aumenta para o dobro de 0,2 para $0,5 \mathrm{mg} / \mathrm{ml}$ na população em geral, mas sete vezes no rapaz dos 16 aos 20 anos.

Existem publicados vários estudos que demonstram alterações das capacidades e desempenho na condução de 0,2 para $0,5 \mathrm{mg} / \mathrm{ml}$ (Figura 4 ).

Existe evidência científica, através de estudos em laboratório (simuladores) e epidemiológicos, que TAS de $0,2 \mathrm{mg} / \mathrm{ml}$ provocam na maioria dos condutores alterações das capacidades psicomotoras necessárias a uma condução 100\% segura. São vários os estudos epidemiológicos que apontam a existência de uma relação forte e directa entre a ingestão de bebidas alcoólicas, a frequência, a gravidade e mortalidade dos acidentes de viação. ${ }^{14}$

Investigações recentes têm demonstrado que, mesmo baixas concentrações de álcool no sangue, ${ }^{15}$ prejudicam 
as capacidades do condutor para a condução, aumentando assim o risco de acidentes. A nova informação sobre a influência das reduzidas taxas de alcoolemia tem conduzido à diminuição do limite legal, medida que, comprovadamente, contribuiu para o declínio da percentagem de acidentes de viação e da mortalidade relacionada com a condução sob o excesso de álcool.

Mounce et al., utilizando modelos de regressão logística, demonstraram a existência de uma relação entre a TAS e a responsabilidade do acidente em condutores que vieram a falecer, verificando-se um aumento da mesma, de forma contínua de 0,0 para $0,5 \mathrm{mg} /$ /ml. ${ }^{16}$ Moskowitz et al., num importante estudo em que procederam a uma muito completa revisão da literatura, através da consulta de 112 artigos científicos, concluíram que as diversas actividades psicomotoras associadas à condução são prejudicadas, de forma diferente, pela ingestão de álcool. No estudo em referência foram analisados aqueles efeitos na divisão da atenção, sonolência, manutenção da direcção, controlo do equilíbrio corporal, manutenção da vigilância, tempos de reacção, orientação espacial, equilíbrio, percepção e diversos aspectos da função visual como a acuidade, controlo dos movimentos oculares, visão periférica, etc. Nos estudos que envolviam simuladores, $73 \%$ dos testes mostram alterações já com TAS de 0,39 mg/ml. Os resultados daqueles estudos indicam que a capacidade de dividir a atenção entre duas ou mais fontes de informação visual ou efectuar tarefas em simultâneo, se encontram já alteradas com alcoolemia de $0,2 \mathrm{mg} / \mathrm{ml}$ sendo que, cada aumento de $0,2 \mathrm{mg} / \mathrm{ml}$ em condutores com TAS positivas, duplica o risco de acidente fatal. É referido por Zador et $a l^{17}$ que " $\mathrm{Ba}$ sed on extensive research over several decades, we now have overwhelming evidence showing that even BACs (blood alcohol concentration) as low as $0.02 \%$ impair driving-related skills". Não existe taxa de álcool no sangue absolutamente segura. Numerosos estudos científicos efectuados desde há algumas décadas (simuladores e avaliações comparativas dos acidentes com diferentes TAS) fornecem ampla evidência que mesmo TAS «reduzidas» $(0,2 \mathrm{mg} / \mathrm{ml})$ podem desencadear alterações significativas nas capacidades sensorial e psicomotora do condutor.

A eventual redução do consumo com a aplicação de TAS reduzidas $(0,2$ ou mesmo $0,0 \mathrm{mg} / \mathrm{ml}$ ) não representaria um prejuízo económico para os países produtores. Em documento de 17 de Janeiro de 2001 a Comissão das Comunidades Europeias é de opinião «que o valor social da redução de acidentes decorrentes de limites mais baixos para a condução sob o efeito do álcool em toda a Comunidade (...) irá muito provavelmente compensar possiveis perdas económicas em algumas regiões». O interesse colectivo, o valor inestimável da vida humana e a evidência científica devem prevalecer sobre o interesse económico de alguns sectores.

Com este enquadramento e no interesse de uma maior segurança rodoviária, a Comissão das Comunidades Europeias recomenda que se caminhe para a imposição de limites de TAS de $0,5 \mathrm{mg} / \mathrm{ml}$ ou inferiores. Recomenda também que todos os Estados-Membros adoptem o limite de TAS de 0,2 $\mathrm{mg} / \mathrm{ml}$ ou inferior para os condutores com menos de dois anos de experiência de condução, para os motociclistas, condutores de veículos de transporte de mercadorias com $>3,5$ toneladas de peso bruto, de autocarros com $>8$ lugares de passageiros e de veículos de transporte de mercadorias perigosas. A EUROCARE (conjunto de 47 organizações não governamentais de defesa e prevenção dos problemas relacionados com o álcool na Europa) recomenda textualmente a adopção dos $0,2 \mathrm{mg} / \mathrm{ml}$ como taxa limite. ${ }^{18}$ 


\section{Qual a Diferenca entre as DIFERENTES BEBIDAS?}

O vinho, a cerveja, os shots, vinho do Porto, Martini, uísque (whisky) são bebidas diferentes entre si na graduação (quantidade de álcool puro que contêm). No entanto, como as quantidades dos recipientes que são servidos são diferentes, considera-se que a quantidade de álcool por bebida servida é aproximadamente a mesma. Ou seja, uma bebida equivale de um modo geral a $0,2 \mathrm{mg} / \mathrm{ml}$.

\section{IDADE PARA BEBER?}

O ideal é não se beber muito antes dos 21 anos. Mas, a partir dos 18 anos, se não se conduzir, se não se beber mais do que duas a três bebidas no jovem do sexo masculino ou uma a duas por dia na rapariga, não é muito grave. Durante a gravidez não se pode beber. Infelizmente em Portugal, é permitido beber a partir dos 16 anos.

\section{Onze Mensagens - Chave sobre}

o álcool e os jovens

1. O álcool é uma droga lícita e o alcoolismo é uma doença

2. O álcool é uma ameaça à saúde pública mundial (OMS)

3. O consumo excessivo de álcool está na origem de 61 doenças

4. Não se deve beber antes dos 18 anos, e não mais de 2 a 3 bebidas por dia no homem e 1 a 2 bebidas por dia na mulher

5. A causa principal de morte e incapacidade nos jovens portugueses são os acidentes de viação

6. O «binge drinking» (embriaguez) é uma das formas mais graves de alcoolismo

7. Uma bebida (cerveja, vinho, ou shot) equivale a uma taxa média de álcool no sangue de $0,2 \mathrm{mg} / \mathrm{ml}$

8. Alcoolemias de $0,2 \mathrm{mg} / \mathrm{ml}$ já provo- cam alterações na condução

9. O risco de morte na estrada duplica de $0,2 \mathrm{mg} / \mathrm{ml}$ ( 1 bebida) para 0,5 $\mathrm{mg} / \mathrm{ml}$ ( 2 a 3 bebidas)

10.No jovem do sexo masculino dos 16 aos 20 anos, o risco de morte na estrada aumenta 7 vezes de 0,2 $\mathrm{mg} / \mathrm{ml}$ ( 1 bebida) para $0,5 \mathrm{mg} / \mathrm{ml}(2$ a 3 bebidas)

11.Em dez anos (1991 - 2001), morreram quase 30.000 portugueses na estrada!

\section{REFERÊNCIAS BIBLIOGRÁFICAS}

1. Cortez-Pinto H, Marques-Vidal P, Monteiro E. Liver disease-related admissions in Portugal: clinical and demographic pattern. Eur J Gastroenterol Hepatol 2004 Sep; 16 (9):873-7.

2. White IR, Altmann DR, Nanchahal K. Alcohol consumption and mortality: modelling risks for men and women at different ages. BMJ 2002 Jul 27; 325 (7357): 191.

3. Marmot M. Alcohol and coronary heart disease. Intern J Epidemiol 1984 Jul;13 (2): 160-7.

4. International Guide for Monitoring Alcohol Consumption and Related Harm. Geneve: World Health Organization; 2000.

5. Hingson RW, Heeren T, Winte MR. Age at drinking onset and alcohol dependence. Arch Pediatr Adolesc Med 2006 Jul; 160 (7): 739-46.

6. Brewer RD, Swahn MH. Binge drinking and violence. JAMA 2005 Aug 3;294(5):616-8.

7. Lewin I. Driver training: a perceptual-motor skill approach. Ergonomics 1982 Oct; 25 (10): 917-24.

8. European Transport safety council. Police enforcement strategies to reduce traffic casualties in Europe. Brussels: European Transport Safety Council; 1999.

9. US Department of Transportation. Traffic Safety Facts, 1998. Washington, DC: US Dept of Transportation; 1999.

10. Carlini-Cotrim B, da Matta Chasin AA. Blood alcohol content and death from fatal injury: a study in the metropolitan area of São Paulo, Brazil. J Psychoactive Drugs 2000 JulSep; 32 (3): 269-75.

11. Redelmeier DA, Tibshirani RJ, Evans L. Traffic-law enforcement and risk of death from motor-vehicle crashes: case-crossover study. Lancet 2003 Jun 28 (9376); 361:2177-82.

12. Comissão das Comunidades Europeias. 


\section{DossiER}

Problemas Ligados ao Álcool
Recomendação da Comissão relativa ao teor de álcool no sangue (TAS) máximo permitido aos condutores de veículos a motor. Bruxelas. Comissão das comunidades Europeias; 2001.

13. OMS. Carta Europeia sobre o Álcool (European Charter on Alcohol). Lisboa: DGS; 1995.

14. Marinho R. Perspectiva médica sobre taxa de alcoolemia de 0,2 mg/ml. Rev Port Clín Geral 2001; 17: 471-86.

15. Moskowitz H, Fiorentino D. A review of the literature on the effects of low doses of alcohol on driving-related skills. Washington, DC: National Highway Traffic Safety Administration (NHTSA); 2000.

16. Mounce NH, Pendleton OJ. The relationship between blood alcohol concentration and crash responsibility for fatally injured dri- vers. Accid Anal Prev 1992 Apr; 24 (2): 201-10.

17. Zador PL, Krawchuk SA, Voas RB. Relative risk of fatal crash involvement by BAC, age and gender. Washington, DC: National Highway Traffic Safety Administration (NHTSA); 2001.

18. WHO Regional Office for Europe. European Alcohol Action Plan. Copenhagen: WHO Regional Office for Europe; 1999.

Endereço para correspondência: Rui António Rocha Tato Marinho Hospital Santa Maria, Av. Prof. Egas Moniz Lisboa 1649-035, Portugal

E-mail: rui.marinho@mail.telepac.pt

\section{ABSTRACT}

There is sufficient evidence to indicate that alcohol is a significant threat to world health according to the World Health Organization. The European countries are drinking too much and one quarter of the young people from 15 to 29 years-old are dying because of excessive alcohol consumption. Portugal has one of the highest alcohol consumptions in the world, beginning in average at 13 years-old. Is also a fact the generalization of high alcohol intake in girls and binge drinking is becoming epidemic which is a particularly dangerous way of consumption.

Alcohol is able to cause 61 diseases, being most relevant in

Portugal alcoholic liver cirrhosis and deaths caused by car accidents, namely in young people. Liver cirrhosis is the tenth cause of death in Portugal and 2/3 of which are alcoholic. Traffic accidents are the leading cause of death in Portuguese young people. Ninety percent of traffic deadly accidents are caused by human error, and half of them are related to excessive alcohol intake. The measures to implement in order to reduce the consequences of the excessive alcohol consumption must consider not only information but also, in order to save lives rapidly, to firmly prevent alcohol intake by increasing the number of breath-tests and stricter the enforcement of the law.

These measures are saving lives in other countries, such as he United Kingdom, where excessive alcohol consumption is a reality.

Keywords: Youth; Binge Drinking; Traffic Accident. 